\title{
Usability Profiling of Enterprise Validation Matrices, Metaphors and Methodologies
}

\author{
Joe Essien \\ School of Computing and Technology, \\ University of West London, St Mary's Road, Ealing \\ London, U.K.
}

\begin{abstract}
The evolution of Enterprise Architecture modeling techniques need for integration of models with other Information Systems collaborations require a methodology that ensures that the heterogeneous models can be validated for their coherence and congruity with common business motivation. However, a major challenge that impedes this requirement is that the more EAF modeling tools and methodologies are broadened, the more complex it tends to become as the tools strive to satisfy all necessary aspects of the enterprise identified collectively. Many enterprises that have adopted a mix and match methodologies are faced with the issue of models consolidation resulting from inconsistent and heterogeneous modeling artifacts over a spurious framework that needs validation. This article seeks to review the composition and capabilities of existing EA validation techniques and to recommend a surrogate innovative approach that addresses specifically model validation. An approach that ensures harmonization of EA models within similar abstractions irrespective of the impediments occasioned by the multi-variant EAF modeling methodologies is proposed.
\end{abstract}

Keywords: Enterprise Architecture, Metamodel, Model, Ontologies, Business Strategy, Validation.

\section{INTRODUCTION}

Enterprise Architecture (EA) has been defined as a practice for conducting enterprise analysis, design, planning and implementation. It is a holistic approach that ensures successful deployment and execution of business strategy. The design of EA consists of coherent principles, methods, models that express the organisational structure, business processes, information and relationship with each other. The major objective of Enterprise Architecture is to provide architectural principles, frameworks, methodologies, processes, tools, knowledge base and techniques that can support the mission of the enterprise. This finery are also expected to facilitate the alignment of artefacts, ensure traceability of relationships, localization, harmonization of interactions and visualization with perspectives in order to make the entire enterprise more productive and efficient. The capability of EA methodology to identify the steps necessary to produce each deliverable of EA development or evolution is of vital importance for validation. This is more so as the practitioner needs to be able to easily determine and execute steps necessary to produce a selected goal or motivation. The consideration of most EA methodology is that it should simplify the EA development and evolution process. For frameworks that deploy a variety of modelling tools, compatibility is important with a broad scope of coverage that supports variety of techniques and technology for process design, methods and repositories. This should be proven pragmatically as complete, concise, and proficient in supporting perspective visualization without complexities.

However, despite that many definitions of EA specify a methodology for accessing, organizing and displaying information; they do not in many expositions include the description of validation capability of the taxonomy produced. Many techniques for validating models are predominantly subjective. In order to correlate the existing multifarious frameworks and make inferences regarding their validation techniques, the descriptions of enterprise architecture framework need to take into consideration the key elements of comparing, correlating and identifying their differences and similarities. Given that the existence of ambiguities and lack of semantic integrity has continued to pose difficulties in implementation of EA modelling and validation, a major benefit of an approach that can offer some kind of consolidation and formalization is that it can advance a precise standard that supports traceability and goal realization. Additionally, it can provide a foundation for alignment of EA abstractions to technological infrastructures, accommodate change, and allow evolving EA modelling techniques to mature consistently with new technologies such as cloud computing, linked data and strategic business transformations. 
Thus this paper is organised as follow; Section two presents the rationale and contributions of this work. Section three presents an overview of commonly used EA frameworks and their capabilities. A general critic and limitations of these methodologies are also discussed in terms of consideration for validation, structure, scope and flexibility. Section four delves into EA validation techniques expatiating on approaches such as maturity matrices, reference models, architecture content framework, balanced scorecard and capability test methodology. Section five presents coherent challenges and critical success factors for validation of EA models. This extends to include communicating their terms and concepts, business model, architectural process, modelling artifacts, traceability, governance, organizational culture, assessment, evaluation criteria and scope. Section six grounds the contribution of this article on Ontology-based model validation and discusses enterprise architecture validation metaphor, model validation patterns, and types of measures for EA validation and boundaries of ontology validation domain. Section seven provides reference to the case study that supports this article and concludes the article by expounding on areas for further research.

\section{CONTRIBUTION AND VALUES}

The major contribution of this paper is an articulate retrospect of approaches adopted by many EAF to validate enterprise architecture models, limitations of these EAF in effectuating validation, challenges of EAF interoperability and advancement of ontologies as a paradigm for realizing validation of EA models. Ontologies have existed for a very long time and has been conceptualised as the basic structure or schema around which knowledge base can be built [1]. Ontologies delineate relationships to structural concepts and define hierarchies bound by predefined assertions. More complex ontologies include axioms that handle increased intricacies in relationships, concepts and constraints desired to bind intended interpretation. Thus as the concern for appropriate representation of content by use of standardised specification languages or other formal logic intensifies, ontologies seamlessly provide the exposition needed especially as it relates to EA models. Therefore, in relation to these contingencies, this contribution presents facets of opportunities that exploit the benefits of ontologies and augments the contribution of this work in the following ways;

i. The exposition of traceability for the Enterprise Architecture artefacts through the use of ontology filters and logical reasoners allows dependencies and effect of change to be more apparent. This innovation apart from facilitating clarity in the presentation of EA model in terms of goals that are required and business artefacts that constitute the processes needed to achieve those goals; it also aids alignment of business strategy with goals as well as determines gaps and overlaps with the EA taxonomies.

ii. Validation of Enterprise Architecture models using ontology querying methodology provides an opportunity for development of new validation semantics that are particularly specific to EA artefacts. Though this would likely be an ongoing heuristic enhancement process for the identification of the most useful generic queries applicable to EA validation domain, the adoption of the approach proposed in this work on more EA modelling projects across divergent establishments would provide further information that can authenticate the generality for the ambience of the vocabulary.

iii. With this supposition, the language semantics is built with preconditions and post conditions applied in a formal way to prognosticate the query for the constraints specified by motivation. Evaluation of the result yields three outcomes; (a) values that allow comparison to ascertain if the tested goal is realized, (b) component traceability and (c) reusable test basis for validation iterations. This validation contribution is systematic, logical, objective and targeted based on input and output artefacts that adhere to set constraints and business rules.

iv. The continuous alignment of business architecture to IT architecture is one of the challenges to the implementation of EA in industry and management of change. The proposition introduced in this work demonstrates that following a transformation workflow from EA model to ontology, unification of enterprise architecture models in particular with unique semantics that allow adept mapping between business and IT architectures can be achieved. This would facilitate the much desired interoperability of heterogeneous EA models through a formalised validation process.

\section{EAF VALIDATION CONCEPTS}

A number of contemporary architectural frameworks are in use today which portends to solve specific needs or concerns of the enterprise. Though some frameworks overlap, address similar views or may even be divested in certain aspects, they still provide a means to implement and integrate the building blocks within the organisation [2]. Several comparisons and analysis have been made between enterprise architecture frameworks [3], based on support and design; Chen et al. [4], based on mappings and relationships, Tang et al. [5], based on high level goals, inputs and outcomes and Schekkerman [6] based on complexity and added value. In this exposition, a different analytic view for commonly adopted EA models is presented based on their composition and structure in order to bring to perspective their validation capabilities and justify the approach proposed in this work.

Considering the myriad of EAFs in use today, a number of methodologies have been identified. These include the Zachman Framework (ZF), The Open Group Architecture Framework (TOGAF), Gartner Enterprise Architecture Framework (GEAF), Federal Enterprise Architecture Framework (FEAF), Generic Enterprise Reference Architecture and Methodology (GERAM), Systemic Enterprise Architecture Methods (SEAM), Dynamic Architecture (DyA), Integrated Architecture Framework( IAF), ISO's RM-ODP, ISO/IEC/IEEE Standards, Department of Defence Architecture Framework (DoDAF) and Treasury Enterprise Architecture Framework (TEAF) and many others. A precursory analysis indicates that the principles, composition, associations, tools and techniques for integrating artefacts of few of these frameworks can actually represent a functional, efficient and harmonious enterprise structural taxonomy as construed by many definitions. These are the ZF, TOGAF, FEAF, DoDAF and SEAM. ISO/IEC/IEEE Standards is considered additionally because it specifies the recommended practice for architectural 
description of intensive systems. These selected EAF or methodologies addressed holistically and more appropriately the elements of strategy, modelling, the overall EA process, methods and techniques, standards and tools that enable the coordination and delivery of the various elements that constitute the Enterprise Architecture within the organization. They also express consideration for goals and motivations. The following subsections therefore describe the implementation of validation within these selected EAFs.

\subsection{The Zachman Framework}

The Zachman Framework (ZF) considered as one of the pioneering models in EA domain is based around the principles of classical architecture that establish a set of perspectives for describing complex enterprise systems [7]. The framework originally referred to as the Information Systems Architecture (ISA) for EA is unlimitedly generic. It can be used to classify broad descriptive representations of EAs thus facilitate the analysis of relative architectural compositions. However, it has been contended that while the Zachman Framework provides a means for organizing architectural artifacts such as design documents, specifications and models, it does not contain concepts that relate to process or methodology [8], hence validation cannot be applied.

The application of the ZF is an extensive and difficult exercise due the large number of cells and complex detailing within the cells. While some of the cells can be modelled using some standard and well-structured techniques, other cells cannot. Realistically, the modelling of some cells in the ZF still remain an open research problem and in particular, well defined modelling language for modelling the technical infrastructures are almost non-existence. There are many issues that are critical to EA modelling that Zachman does not address such as step-by-step process for creating an architecture or guidance in assessing architecture's appropriateness or usability. Additionally, the relationships between the different cells that make up the framework are completely ignored. As heterogeneous modelling techniques are used to populate each of the cells including sub details within the cells, it is impossible to adopt common or even identify similarities across the cells in order to allow the mapping of relationships. Thus the fundamental basis of the $\mathrm{ZF}$ is the segregation of the enterprise into isolated units.

Rather than promote the development of multiple views of EA based on stakeholders concerns, the ZF assumes that there can be only six discrete viewpoints achievable with six roles namely planner, owner, designer, builder, programmer and user [7]. With non-specification of hierarchical levels across the rows that distinguish the viewpoints, symmetry or alignment cannot be realized. The ZF presents contemporary concerns such as security, governance, validation, artefact orientation and change management. Since enterprises evolve, these deficiencies make the ZF distinctively incapacitated as a prescriptive framework.

It has been maintained that though the ZF is fashionable and a conviction of conglomerate affinity, it is founded on a subjective, untested observation thus lacks scientific foundation [9], [10]. As many tools can be used to depict structural components in ZF, it is difficult to implement validation as the diverse tools used cannot offer a consistent component description for relating all the objects across the different layers of the framework.

\subsection{The Open Group Architecture Framework}

The Open Group Architecture Framework (TOGAF) is created and maintained by The Open Group (TOG). It is built based on an earlier framework known as Technical Architecture Framework for Information Management (TAFIM) originally devised by the U.S. Defence Department in 1995. Over the years, several versions of TOGAF have evolved making it increasingly comprehensive and adaptable. Due to this maturation of TOGAF in terms of structural composition, reliance on modularised and standardised existing proven technologies, it is most widely accepted as an approach for designing, planning, implementation, and governance of enterprise information architecture. TOGAF is modelled at four levels to encompass Business, Application, Data, and Technology aspects of EA [11]. The core TOGAF contains descriptions of an Architecture Development Method (ADM) and is related to other techniques specified in its Architecture Content Framework (ACF), Enterprise Continuum (EC), TOGAF Reference Models and a Capability Framework amongst other enhancements. A more detailed description and updates on TOGAF are available at their portals.

However, there are also pitfalls associated with TOGAF. One of such is the attempt during implementation to execute every phase, deliver each artefact and create all repositories defined by TOGAF. Though this in itself may not be wrong, TOGAF strictly emphasizes as key for success, though not explicitly, the need to make selections, and tailor the framework to the context at hand so as to optimize the generation of real business value. Another limitation is that in many domains, TOGAF has been adjudged to be very technical-minded and focused on delivery of models although architects need models, technology, instruments, languages, and deliverables to effectively communicate with stakeholders, TOGAF is not wholly specific with respect to generation of documentations. In fact, it provides very little in the way of prescriptive document templates [12], [13].

With respect to validation, though TOGAF is incorporated with the ACF as means that expresses content metamodel to provide a definition for all the types of building blocks that may exist within architecture, it has been suggested that the ACF is not flexible enough to adjust to the different contexts found in the organizations. The ACF represents the whole enterprise and that is too much information. In order to obtain effective communication with the stakeholders and participants, the architecture contents should be presented in views that address the particular concerns of each interest group. Thus it has been argued that the ACF is inadequate as a means for validating, measuring and communicating the impact of TOGAF implementation. 


\subsection{Federal EA Framework}

When compared with some renowned contemporary methodologies, the Federal EA Framework comprises of a comprehensive taxonomy like the ZF and an architectural process, like TOGAF. The FEAF shows clear collaboration with the ZF on three of the six columns (what, how and where) while the remaining three columns (who, when and why) are not considered. Though these three collaborations correlate with the three significant aspects of the $\mathrm{ZF}$, unlike the $\mathrm{ZF}$, the constraints of each perspective are additive. In other words, the constraints of higher rows affect the rows below though the reverse is not necessarily true. As the FEAF is additive, there is a risk of making illogical suppositions if all cells are not modelled. Five FEA reference models are set to establish standardisation of a common language in the FEAF. These consist of a set of interrelated references designed to promote cross-agency analysis and the identification of duplicative investments, gaps, and opportunities for collaboration but not the models. Collectively, it has been claimed that the reference architecture describes important elements of the FEA in a common and consistent way that facilitates communication, cooperation, and collaboration across political boundaries.

However, when the five FEA reference models are considered in terms of their validation capability, it has been pointed out that the FEAF is too flexible. As it allows individual federal agencies to use methods of choice, varied work products, and tools to define their own EAF [14], validation of the EAF in a consistent way is distinctively impossible and impracticable. Consequently, the FEAF and its Reference models are evolutional and cannot be applied comprehensively for many other domains.

\subsection{Systemic EA Methods}

Systemic Enterprise Architecture Methods (SEAM) is a family of methods for strategic thinking, Business / IT alignment, and requirements engineering. The originality of SEAM is embodied in its ability to integrate generic system thinking principles with discipline-specific methods [15]. In contrast with other frameworks, SEAM has the capability to relate different disciplines through common systemic principles thus represent systematically business, organizational and IT concepts through a commonly shared modelling ontology. This advantage leverages its susceptibility to acquire specific knowledge by use of shared vocabulary and heuristics of each integrated discipline [16]. The family of SEAM methods are comprehensively explained in the works of Golnam [17].

However SEAM has been criticised as being concentric on functionality analysis with emphasis on cost and security while other dimensions such as technology, business behaviour, knowledge and information management are largely ignored [13]. Additionally as SEAM places emphasis on the properties of these built functional models and not on the expertise and process for modelling, the use of divergent tools for modelling its architecture in this context actually perpetuates the exponentiation of complexities. Compared with many other frameworks and methods, the SEAM brings an elaborate analysis of the environment based on Reference Model for Open Distributed Processing RM-ODP approach. It attempts to provide a systematic ontology for system modelling with the ability to integrate the whole of EA coherently. A point of view in favor of the SEAM approach is that the same concepts and principles can be leveraged to model business, operational and IT aspects simultaneously. Thus contextual modelling of processes seamlessly interrelates with modelling of behaviour, segment and goal.

In practice, SEAM is used to scope projects. Though Enterprise Architecture Frameworks are structured in hierarchies that allow analysis across different aspects and layers, SEAM does not place significant emphasis on technology in its taxonomy [15]. In the well cited article and work presented by the renowned originator of SEAM [15], there is no discussion on how models created with the SEAM can be validated except for a passive reference that since the SEAM methodology is iterative, in adapting the model to represent changes within the organisation, validation and testing can be achieved with real people against the hypothesis made in the model. In a prospective case study of [18] where an enterprise architecture methodology for business-IT alignment is implemented from adopter and developer perspectives using the SEAM methodology, SEAM validation is effected by determining the magnitude of relationships that exist between constructs and formulation of intensity indices for each construct based on questionnaire instrument. This is analogous to the use of balance scorecard method.

In an attempt to validate the output artefacts of the SEAM, in a recent work of Golnam [17] which included the works of Wegmann [15], a problem structuring method (PSM) called "Value Map" is introduced. Value Map was designed to be an extension to the Supplier Adopter Relationship Diagram in the SEAM and aimed to assist in understanding, analysing and design of value creation and capture in service systems. To validate the usefulness of the Value Map in SEAM, an empirical study was also conducted to demonstrate that the Value Map can help business practitioners in understanding and analysing customer value, customer value creation, and the value capture processes. However, the work clearly contradicted its aim as it emphasizes that the Value Map does not validate the model artefacts after all but provides only a graphical representation of value creation and capture concepts.

\subsection{Department of Defence Architecture Framework}

The Department of Defence Architecture Framework (DoDAF) is an architecture framework for the United States Department of Defence (DoD). Organized by viewpoints, it consists of a large number of systems architecture frameworks and provides 
visualization infrastructure for the development and documentation of all major U.S. DoD weapons and information technology systems. While DoDAF is clearly aimed at military systems, it has broad applicability across the private, public and voluntary sectors worldwide [6]. The foundation ontology for the meta-model in DoDAF is defined by the DoDAF Meta-Model (DM2) and consists of conceptual data models, logical data models and physical exchange specifications. This underpins the DoDAF framework and defines the types of modelling elements that can be used in each view and the relationships between them [19]. Consequently, the views of DoDAF distinctively define artifacts for visualizing, understanding, and assimilating the broad scope and complexities of an architecture description through tabular, structural, behavioural, ontological, pictorial, graphical, probabilistic and conceptual means [19]. This makes DoDAF specifically suited to large systems with complex integration and interoperability challenges as it provides common denominator for understanding, comparing and integrating architectures across organizational and multinational boundaries.

In consideration of the approach adopted by DM2 in the validation of its models, DM2 establishes and defines vocabulary constraints for linguistic context and description for DoDAF models as applied to the six core processes. It specifies the semantics and format for federated EA data exchange between architecture development, analysis tools and architecture databases across the DoD Enterprise Architecture Community of Interest (COI). Furthermore, DM2 supports discovery and lucidity of EA data using DM2 categories of information and precise semantics augmented with linguistic traceability [20]. Consequently it is widely acclaimed that though DM2 provides a basis for semantic precision in architectural descriptions and supports heterogeneous architectural description integration and analysis, it does not substantiate the model's artefacts. In practice, DoDAF deploys very substantial levels of details. There is no clean separation between the planning and development stages and, as a result, there tends to be substantial duplication of effort between development and planning. Though DoDAF has a respectable pedigree, many practitioners do not have a clear understanding of its scope, including how the models can formalized, levels of interoperability and what types of validation or reference architecture can be applied.

\section{EA VALIDATION TECHNIQUES}

The success and quality of EA is influenced by several interrelated factors. While the challenges faced in validating EA seem to be dependent on commitment and communication through a common language, it also appears that if the EA objectives are well defined to support the business objectives, it would be easier to gain both the top management commitment and the organizational subscription. Though it has been argued that the adoption of metaphorical measures for modelling EA can guide in the selection of important critical success factors for its validation; and many enthusiasts of EAF methodology claim to abide by some formalized precepts, differential analysis indicate that many EAF do not embellish in their process a validation approach for produced artefacts. In many cases, reference models are deployed such as in TOGAF, FEAF and DODAF. Reference models for instance by themselves do not annotate or correlate output artefacts but are merely used to affirm externally goals realization based on subjective dimensions that pertain to the implementation domain. To be systemic and pragmatic, a reference model should include a clear description of the problem that it portends to solve, and the concerns of the stakeholders who need to see that problem solved. The following subsections present contemporary validation approaches for EA and their limitations.

\subsection{Maturity Matrices}

Maturity Matrices are used as an instrument for assessing the level of EA development in organizations. Often, it is a list of key areas that represent different dimension within the EA. Various levels for control of systems maturity have been proposed in EA. In some cases this necessitates outright extension of the frameworks such as in the TOG consortium with the introduction of Architecture Content Framework (ACF) and others by insertion of principles that facilitate validation such as in FEAF; where assessment frameworks with reference models are used. Maturity Matrices have also been adapted in many EA implementations with autonyms such as Dynamic Architecture Maturity Matrix (DyA MM), Capability Maturity Matrix (CMM), Risk Maturity Matrix (RMM), Test Maturity Matrix (TMM).While some implementations of maturity matrices have been considerably simple, many others have been adjudged complex, permeable and inapplicable in many scenarios for validating EA.

However, one major disadvantage of the maturity matrices is that the prioritisation of the key evaluation criteria related to specific identified goals and concerns of the business are absolutely subjective [21]. The graduation along the levels of maturity scale as a means of determining progression can be elusive. Occasionally, management do compromise logical accomplishment of high valued goals and adherence to constraints in favour of the resolution of immediate propositions. Also, a productive iteration with a high strategic sequential value may be placed on hold while its resources are assigned to a minor concern in order to boost the result of scale of progression on the maturity matrix. Indeed, it has been argued that the assertion of the level of maturity of the EA is often based on cognitive opinions gathered through hypothetical compendium thus it is possible that the questionnaires may not always be well understood by the respondents. Consequently the questioner's preferences may influence the outcome in given circumstances [22]. This bias often rescinds the validity of the outcome of maturity matrices.

\subsection{Reference Models}

Many enterprises use the Reference Model (RM) as an abstract framework consisting of interlinked set of defined concepts to encourage clear communication between EAF. The reference model represents a complete set of the component of the EAF as specified from the business functions to system components, and is used as a frame of reference to communicate ideas among 
components and an indication of their relationship. Specifically a RM creates the standards for both the objects that constitute the model and the relationships to one another. Applied in validation of EA, a RM describes a set of business measurements needed to create balanced scorecard. Each measurement is assigned to specific business roles that allow allocation of responsibilities for production of quality output.

Though RM are preferred by many EA practitioners as a methodology for assessing enterprise maturity, it has been asserted that Reference models are not comprehensive enough as means of validating EA models due to its inability to describe the archetypes that occur in an EA environment. Additionally, since the list of entity types and constraints defined by RM must adhere to some Reference Architecture, it cannot provide enough information to serve as a reference metrics for the entire framework in many cases. Thus the usefulness of a RM is limited as it is to the assumptions about the expected maturation of the business and technology platforms deployed in a particular domain. As an effective validation approach is expected to support the process of envisaging and simulating a variety of pragmatic test scenarios specified by motivation, this is not provided for by the RM.

\subsection{Architecture Content Framework}

TOGAF is an example of architecture with content categorization. As one of the most popular frameworks, it provides a uniform representation for diagrams to describe its enterprise architecture models using ArchiMate. ArchiMate Enterprise Architecture Modelling Language is developed to support TOGAF Architecture Development Method (ADM) and offers an integrated architectural approach that describes and visualizes the different architecture domains [11]. This encompasses both the underlying relations and their dependencies [11]. In response to the need for validation and testing the effectiveness of EAF, TOG introduced version 2.0 of ArchiMate with an extension incorporated with tools; first to model motivation and secondly to assess the Architecture Content Framework (ACF). Motivational concepts are used to model the intentions and reasons that underlie the design or change of the enterprise architecture. Motivations influence, guide, and constrain the design thus allow validation to be performed on the model.

TOG claims that the ACF defines the various models that describe a generic EA as its coverage includes EA artefacts and definition, processes, standards and guidelines for artefact development and the associated modelling notations that enable common understanding and collaboration. But specifically, the very core of ACF is a concept that defines a set of content specification that is coherent with the four major dimensions of its cognate modelling language ArchiMate; namely business, application, information and technology with selection and customization driven by motivation. While many EAF continue to use maturity matrices as the practical assessment instrument for identifying gaps between business vision and business capabilities, the ACF is a significant innovation of TOGAF designed to provide a structured metamodel for architectural artifacts with support for checklist of architectural outputs.

TOG claims that the ACF appropriated with consistent architecture building blocks, allows for better integration of architectural work products and provides a detailed open standard for describing architectures [23]. However this has not been exhaustively proven as the assessment methodology is not integrated with ArchiMate Core itself. In addition to the ACF, Maturity Matrices still play an important role in TOGAF to identify the level of compliance between business vision and business capabilities.

\subsection{The Balanced Scorecard}

The Balance Scorecard is a strategic planning and management system that is used extensively in business, industry and government to align business activities to the vision and strategy of the organization. The objectives of Balance Scorecard are often extended to include the improvement of internal and external communications and monitoring of organizational performance against strategic goals. Though the Balance Scorecard provides a framework that facilitates performance measurements, its ability to help planners identify what should be done and measured has been contested [24]. In most implementations, the Balanced Scorecard is categorised into four perspectives to present learning and growth, business process, customer view and strategy mapping. Therefore the development of its measurement metrics is also done by analysing collected data relative to each of these perspectives [25]. In this respect, Balanced Scorecard passably assists organizations in clarifying their financial vision, strategy and helps to translate them into action.

Many frameworks use a checklist for balanced scorecard. Though these may achieve some levels of comprehensiveness when initiating EA, it still potentially amounts to benchmarking of expected functionalities or outcome of desired process and not the validation of the models or artefacts of the EAF. Thus at best, the checklist helps to determine targets for which EA evaluation criteria, metrics and methods could be developed with respect to business behaviour from different perspectives of the EAF.

Nevertheless, the Balance Scorecard is prone to many limitations. In practise, several assumptions are made in the evaluation of its process and outcome. For instance it is common to assume that everyone understands the terminologies used; that the organization's strategy has been correctly formulated by management and that the business plan is the right one. However, it has been proven that these assumptions are not always flawless [25]. Another limitation is that of the expected large number of participants in order to ensure that all areas are represented. The need for this is based often on a conscious attempt to try to meet the objectives of every participant's expectation and to ensure that their extensive knowledge is harnessed. In many exertions of the approach, it has been affirmed that the Balanced Scorecard can be very subjective; based strictly on qualitative scrutinisation. 
For this reason, the Balanced Scorecard has been perceived as unsuitable for validation of model as it does not relate model artefact, relationship to motivation distinctly.

It is also argued that when validating scenarios such as presented with traceability within the EA domain and relationships amongst model artefacts the balanced scorecard method does not make sense [26]. It has been suggested that if the scorecard fails to include financial and non-financial objectives, it loses its value as a strategic tool. The balanced scorecard must be continuously updated to reflect changes in the organisation. This requires time, resources and labour which could act as a limitation for smaller organizations without commensurate visible added value.

\subsection{DoDAF Capability Test Methodology Approach}

To enhance the effectiveness and efficiency of the DoDAF through capability assessment and evaluation, innovative enterprise initiatives were undertaken within the Department of Defence (DoD). A key competence that specified the enterprise-level Capability Test Methodology (CTM) was developed to deliver joint capability assessments and evaluations across the acquisition life cycle of DoDAF by the Joint Test and Evaluation Methodology (JTEM). Primarily, the endeavour purposed to identify gaps, seams, and overlaps related to testing in a joint environment of DoDAF. Intensive documentation with respect to process anomaly in policy, organizational or resource application, changes outside the test scope is a crucial part of this approach.

However, there had been constraints identified with the extended DoDAF which inhibits the potentials of these enhancements and its capabilities. For instance, it has been contested that DoDAF models use sporadic and incongruous CTM templates to describe essential CTM concepts including joint mission concepts, measurement metrics for metamodel and model performance, task performance, and goals actualization levels [20]. Therefore the taxonomy is structurally deficient. Another critical deficiency identified is poor integration of test and evaluation measures in relevant DoDAF model and the CTM test plan test matrix. Though DoDAF artefacts are found to be relevant when creating the CTM's Joint Mission Environment (JME), discrepancies are noted between the DoD artefacts and the model design methods [20]. Gaps are also identified when comparing the CTM's evaluation business rule structures, referenced as the Capability Evaluation Metamodel and DoDAF's data model, referenced as the Core Architecture Data Model.

\section{CHALLENGES AND CRITICAL SUCCESS FACTORS WITH VALIDATION TECHNIQUES}

A high quality EA model must conform to some agreed, understood business requirements, motivation and governance processes to guide its design and validation. Critical Success Factor (CSF) stipulates the desired attributes that need to be ascertained in order to assure that quality of EA model. It emphasizes those considerations that must be addressed exceedingly well in order to attain EA maturation. Maturation in this case refers to the organization's capability to manage the development, actualization of business goals and ascertainment of constraints of EA models. With respect to EA, CSF focuses on measurable indices that can be effectuated to ensure effective validation. A more articulate discourse of these challenges and CSF is thus presented.

\subsection{Communicating EA Terms and Concepts}

Though common and well-defined vocabulary of terms and concepts has been identified by some practitioners [8], [27], there is need to uniquely define and document the key architectural concepts with sources in which the model is based. This is necessary due to challenges that often emerge as a result of poor communication or specification of adopted plans and strategies [28]. The means of various communication channels and the timing, phases or situations in which the communication relates to the architecture are often not stated [29].

\subsection{Business Model Driven Approach}

The primary approach to the development of most EA is through consideration of the business processes while adopting a model driven approach. This is envisioned to establish that EA initiatives are traceable to the business strategy and alignment between business and IT [6], [30]. The challenge here is how to determine that the business strategy and related business requirements are taken into account in the design of the architectural model. The techniques for recognition and documentation of the business requirements for the architecture are paramount to the appropriate specification of the framework, definition of views and levels of abstraction.

\subsection{Architecture Process for Model validation}

This involves the application of appropriate processes for the design of EAF. The challenge of identification of an adaptable analysis to be adopted, constructs, constraints and theories expedient for modelling predefined viewpoints of an EA has been identified as a problem that inhibits the validation of models and associated artefacts in the works of Morganwalp and Sage [31], Stanley and Uden [32]. In many instances, there is lack of guidance for the architectural decision making and documentation process. This includes documentation of the support for reuse of the processes, instructions, models or other artifacts. As variant visualization techniques, modelling languages and support tools have been adopted over the years in many organisations to model 
EA, the issue of business and Information Technology alignment continue to be one of the most relevant concerns in these organisations. Therefore, since EA and Information Technology have different and distinct governance approaches, many practitioners have proposed and emphasized that there is need to establish a common frame of reference in methodologies and processes adopted [28].

\subsection{EA Models and Artifacts}

As the models and artefacts are valuable in the communication of the architecture to the various stakeholders, it is important that their definition and documentation be defined extensively enough to convey the appropriate meaning to all stakeholders. Models provide a coherent and concise picture of the enterprise thus need to be communicated to relevant stakeholder in a clear and comprehensible manner indicating the relevant views, composite artefacts and dependencies. The models should also address the current situation (as-is descriptions) and the future situation (to-be descriptions) in conformity to the architecture principles and standards [33].

\subsection{Enterprise Architecture Traceability}

One of the responsibilities of the Enterprise Architect is to provide complete traceability from requirements analysis and design artefacts, through to implementation and deployment. The formal definition of traceability usually refers to the ability to link requirements to stakeholders' rationales progressively to corresponding design artifacts, code, and test cases. Thus Traceability is intended to support numerous EA activities such as change impact analysis, compliance verification, constraints testing and requirements validation. However in EA, traceability often means different things to different people. Some practitioners refer to enterprise model traceability as prove for alignment to business goals; end-to-end traceability to business requirements and processes; a matrix that maps systems functions back to operational activities; reference across artefacts such as services, business processes and architecture; a footprint between a technical component and a business goal. Traceability has also been used to imply identification of associations between artifacts from business and IT strategy to solution development and delivery. However, despite these divergent perceptions of the bounds of traceability, there is a general concession that by adopting traceability between business, information, applications and technology architectures, it is possible to evaluate the EAF against operational performance and business needs to determine areas where misalignment occur and change needs to take place. Unfortunately the practice of constructing and maintaining traceability especially in the form of a matrix is that it is very arduous and over time the traces tend to erode into an inaccurate state unless date/time stamped or versioned.

\subsection{Enterprise Architecture Governance}

Governance and management have been given various explanations depending on the context. Though in general, governance denotes the management and organizational aspects of architecture [34], it can also infer to the principles that guide an organization to make decisions, set priorities, allocate resources and manage its architectural processes [35]. Some key questions related to EA Governance are as follows: Is the architecture governance structure defined, documented and complied? Are the roles, responsibilities and authorizations defined, documented and complied? Are the processes, activities or tasks (such as definition of the architecture policy, principles or architecture compliance strategy) defined and documented (Control Objectives for Information and related Technology, 2000? [34]. Thus there is a challenge in maintaining effective governance processes and activities, identification of risks and management that is needed for validation especially when adopting the maturity matrices approach. The extent of integration of the EA governance processes to the organization's business management processes, such as strategy refinement process is also an issue often underestimated. This is because EA development is usually conducted through projects and project management skills but does not essentially include validation as a crucial concern in order to assure the success of the project.

\subsection{Organizational Culture}

While developing an EA, the organizational culture should also be taken into consideration to achieve good organizational and cultural fit [36]. In many cases cultural changes are inevitable especially in the development and adoption of EA. Organisational culture includes aspects such as attitudes towards changes by stakeholders, in the communication environment, technological innovations and economic dynamics. The organization's structure also has serious impact on the success of an EA. Thus there is need for attitude towards EA to be focused on as an approach that can guide the business and IT decision making processes rather than as an auditing or controlling mechanism [34]. A trusting organizational culture facilitates open communication, interdepartmental interactions, objective evaluation, and constructive criticism and helps to improve the overall EAF [29].

\subsection{Assessment, Evaluation Criteria and Scope}

Engagement in EA assessment and evaluation is often considered as part of the EA governance [31]. EA evaluation is challenging because its effects and consequences are often realized much later during the life cycle of the organization's endeavours. Issues in EA evaluation, planning and implementation have been identified as concerning the models and artifacts; processes, maturity strategies, value, goals and principles; business-IT alignment, effectiveness, completeness and correctness of the EA. Other issues 
include utilization and usage of architectures; people's competency and skills; work environment including culture, leadership and structure. Thus defining the scope of the purpose of EA artefact assessment and evaluation has also been identified as a challenge [37]. Determining the evaluation process and criteria, how and when the evaluation is conducted has been an issue. In most cases, validation platform, empirical data, metrics and tools are not formalized making the entire process not only complex and intricate but difficult for generic, formalised or iterative approaches to be developed.

\section{ONTOLOGY-BASED MODEL VALIDATION}

Though the need for an approach for evaluation of ontology development has grown steadily ever since its debut [38] and a number of ontology transformation, integration methods and tools exist, no global and comprehensive approach for the concern as it relates to EA models has been proposed to date. As it is anticipated that ontologies would be a crucial components in the leverage of other technologies such as cloud computing, big data and change management, the concepts of development of semantics able to cope with interconnectivity of semantics has continued to arouse significant interest. The lack of well understood and shared notions of ontology evaluation and validation has also significantly slowed down the transition of ontology from esoteric symbolic structures to reliable enterprise postulate. Several studies conducted to present a formal approach for ontology evaluation and validation identified three main types of measures. These are categorised as structural measures typical to the ontologies presented as graphs; functional measures related to the intended application of the ontology and of its components; and usability profiling measures which specify the level of annotation of the considered ontology [38]. The application of ontologies to validate model structures and its connotation has not been disputed as capable of ensuring the retention of the domain-specific quality of the model. The satisfaction of domain-specific requirements of the model represents the particular motivation within the domain of interest.

In practice, ontology is evaluated as a diagnostic task based on ontology descriptions for models [39]. In this work EA model transformation to ontology is proposed as there has been no specific literature that claims this methodology of profiling EAF for the purpose of validation. Description of models for ontology validation subsumes explicit knowledge about the model artefacts and is critical to the evaluation of the ontology based on structure, role and function of the considered ontology. Parameters for the description typically denote the quality of the ontology and are construed in accordance to a preferential hierarchy. In validating ontologies, issues considered during development include (a) the capability of the ontological categories to be grouped according to some criteria; (b) the relationship between the ontology category elements and (c) formalization of the visual model in a stereotyped notation that is understandable to the stakeholder. This enables the process of identifying variances especially between heterogonous composites for the model by comparing structures, objects and compliances and use of heuristic methods which extends the rules. This allows the inference of conclusions from imperfect or incomplete patterns.

One of the established methods of evaluating quality of artifacts in otology is to develop a quality model usually done during the early stages of the ontology development, and serve as guidance throughout the project [40]. This is synonymous to our approach where quality model can be synthesize or derived from motivation. Ontologies are developed only through transformation in this case from EA models which target parameters throughout the development, incorporating validation attributes that facilitate testing. Thus the ontology would contain patterns of qualitative and quantitative measurements of various aspects. In the case of EA this is identified as goals and constraints. The quality of ontology is sometimes measured across two dimensions: its accuracy and its comprehensiveness [40]. Almost the entire range of standard testing techniques used in programming consistency, integrity, validation, redundancy can be applied to test the validity of ontology. A good summary of quality evaluation criteria for ontology can be found in the work of Stvilia, [41].

\subsection{Enterprise Architecture Validation Metaphor}

An important and common dilemma that intrigues the disparate methods adopted currently for validating and assessing EAF is how to systematically seek information within the framework [23]. Evidence suggests that the most ambivalent information is that which pertains to the rationale for the design decisions taken for any model [42]. The design rationale confers the justification behind the nature of the validation approach and the reasoning that goes into determining the artefacts of the model that should be validated. Thus capturing design characteristics enables its retrieval, enhances significantly the artefacts integrity and increases the effectiveness of the validation approach. Similarly, the design rationale also supports traceability and promotes collaboration amongst design artefacts, exposes differing EA viewpoints and facilitates integration of model abstractions. For Validation Extension for metamodels, the design decision which delineates the validation artefacts involves contemplation of the following capabilities;

Capture: The ability to capture information through the process of mental filters or guides using for example the VPEC-T [43] thinking framework would prevent loss in translation from business needs to IT solutions. VPEC-T is most suitable for use when analysing the expectations of multiple parties having different views of a system in which they all have a common interest but with different priorities and responsibilities.

Formalization: The proclivity to transform models with its motivation and constraints in a formalised way is a capability that allows the exposition of desired validation dimensions in ontology. There are many ways in which metamodel argumentation may be structured. Common thought preferences include the use of schemas having different stakeholder perspectives, constraints and principles, goals representation and key drivers. 
Relationship Retrieval: This disposition provides a process for artefact traceability and collaboration. The association between artefacts in this way elicits knowledge encapsulation upon transition.

Characterisation of validation artefacts: To exert the archetypes of validation artefacts within EAF, a stereotype can be formalized with measurable metrics from extensible EAML such as the ArchiMate modelling language. To allow correlation with motivation, it is recommended that such extensibility should aim to transverse all artefacts of the Business layer as this would enable the extended viewpoints to be precise and independent of each other. The augmentative formalization also would allow concepts and constructs that span the entire EA framework. This makes the conception well suited for cascading of measures throughout the ontology and collaboration of dependencies.

\subsection{Model Validation Patterns}

Much literature on ontology evaluation focus on generic functionality dimensions rather than structural composition. Based on the principles for specifying model validation rules, predefined criteria or constraints are rarely used in the process of EA model validation [23]. Often validation is considered after the models have been developed or integrated by assessing through subjective means its appropriateness of the semantics underpinning the decision-support. In the context of EA, this informal verification to specify the act of proving or disproving the correctness of intended model design and logic underlying a metamodel and model with respect to motivation or constraints is incommensurate. In EA, the theoretical principles for validation rules specify two integral levels of validation which ensures comprehensiveness [23]. These are the active and passive levels. These rules applied in EA enhance the quality of its artefacts and produce a more unified validation methodology applicable across all layers of the architecture. Classification into these two levels also allows parallelism to be grounded into the validation process resulting in a thorough objective test. The graphic representation of information within these levels uses direct contiguous relationships which facilitate cohesive synonymous visualization of abstractions across the derivative model instances.

\subsubsection{Types of Measures for the EA Validation}

Ontology evaluation of EA model artefacts deals with establishing their qualitative dimensions and relates specifically to Goal Realization, Perspective Visualisation, Behaviour Analogy, Model Traceability and Constraint Assessment within the model. In answering the question "What should be validated in ontology to establish its definitiveness with derivative EA model", validation themes are defined as a set of motivational specifications for the components or aspects of the model to be tested. The validation element modelled with the EAF core defines the metrics, what types of test are to be carried out on the components and the expected results. Test attributes are specified at this level. Constraints expressed within views and perspectives incorporate principles that guide semiotics of the test basis construct. This stage is iterated to develop the various viewpoints such as process and functional models mapped into the ontology. Traceability amongst the other components is established by exerting associated relationships. Evaluating the result yields three outcomes; (i) output abstractions that allow comparison to ascertain if the tested goal is realized, (ii) Resource Description Framework Schema that provides the construct to executing queries and (iii) Resource Description Framework graphs that facilitate traceability.

Using this approach, the quality of the ontology may be assessed relatively to these various dimensions with the structural dimensions represented as a graph. The functional dimensions which are related to the intended motivation of a given ontology and of its components can also be validated by use of ubiquitous query language on the Resource Description Framework (RDF) such as SPARQL. The usability related dimensions for validating the functionality on the ontology would obviously depend on the level of annotation and heterogeneous measurement metrics applied during mapping.

\subsubsection{Boundaries of Ontology Validation Domain}

The boundaries of the knowledge domain addressed by ontology validation are determined by validating the ontology according to its fitness to an existing knowledge repository. This not only makes the validation dependable at design-time and reuse-able but subsumes related data available with the model. Compared with other methods of EA model validation discussed earlier which can be described as a black box approach with a predominant requirement of external rational agents, the proposed approach using ontology deploys explicit knowledge base consisting of the internal structure of the model. It consists of data sets that are exclusively used to sample the representative knowledge repository of the model. On this basis, the internal structure of the model is well encapsulated and forms a benchmark for comparison with expected results or motivation. The boundary of validation also permits assessment of usability profiling of the model as the knowledge repository of the ontology consists of relationship dimensions and annotations of the model necessary for traceability.

Annotation provides the necessary extension for information, business and structural aspects of the EA business layer. These three basic segments of usability profiling relate to the Goal Realization, Perspective Visualisation, Behaviour Analogy, Model Traceability and Constraint Assessment as shown in Figure 1. The Model Traceability determines whether the artefact within the information aspects required for the actualization of business behaviour is available and can be traced to motivation specification. Perspective Visualization asserts that the perceptibility of the accessed artefacts is in consonance within specified privileges, roles and interfaces of the structural aspect of the EA. Business Behaviour Analogy is associated with relationships amongst artefacts and their ability to function as expected in normal and unusual situations when triggered by business events. Constraint 
Assessment validation determines whether a component meets some specified constraints stipulated for actualizing desired business behaviour; and Goal Realization assesses to what extent the intended business goals are achieved in relation to either the actual validation outcomes or impacts on other components.

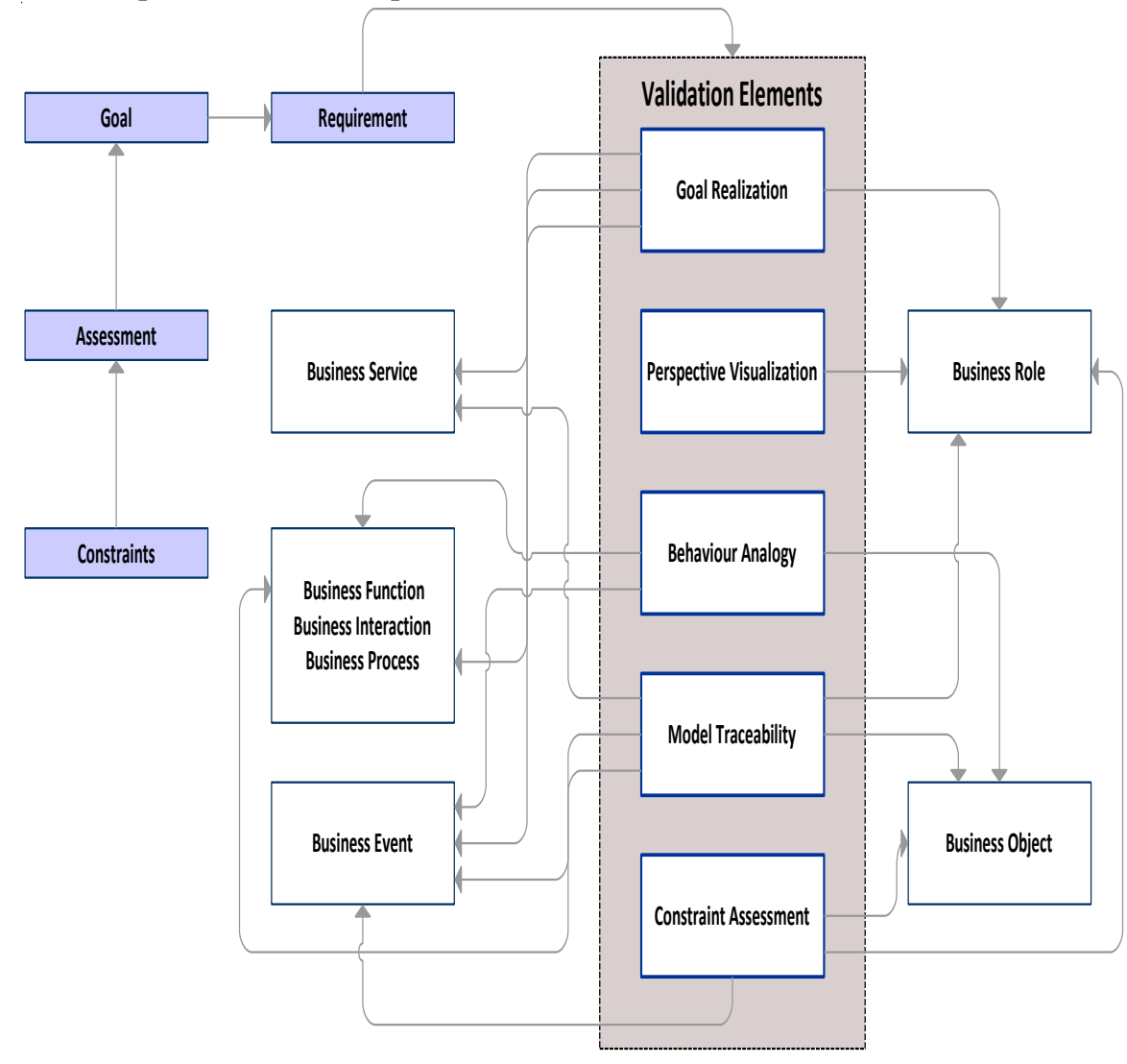

Figure 1: Usability profiling of Validation metrics

The boundaries and extent of validation differ depending on the purpose of the design from motivation to direct EA model, maintenance of metamodel or even the abstract meta-metamodel. As such validation with ontology may not necessarily establish the full connotation and traceability as expressed by their metamodels and frameworks. The efficacy of this approach depends on the proclivity to carry out meaningful formalization before transformation in order to maintain the preciseness of semantics and principles from domain specific constructs to unambiguous descriptions of their concepts in the ontology.

\section{CONCLUSION AND FURTHER RESEARCH}

Ontology validation is exemplified in the works of Oussena \& Essien [44], a research that supports this article. One of the outcomes addressed the definition and formalization of metrics that allowed measurement of different degrees of conformity to motivation to be achieved. Values of the proposition as expressed in the models are presented from varied perspectives and transformed to ontology for validation. However, there is need for the syntax and semantics used in the approach to be enhanced through a more rigorous implementation. This would lead to the development of more principles and patterns for designing and validating architectures for various purposes such as interoperability, flexibility, modularity and goal actualization. It would also provide the grounding for future architecture development and validation on a more scientific basis. Thus there is also an important need to develop an agreed architecture representation language and evaluation method/metrics so that architecture proposals can be properly described, assessed and compared. This can drive the vision for further research to support EA model validation.

\section{REFERENCES}

1. Uschold, Mike, and Michael Gruninger. "Ontologies: Principles, methods and applications." The knowledge engineering review 11.2 (1996): 93-136.

2. Schekkerman, Jaap. How to survive in the jungle of enterprise architecture frameworks: Creating or choosing an enterprise architecture framework. Trafford Publishing, 2004.

3. Iyer, Bala, and R. Gottlieb. "The Four-Domain Architecture: An approach to support enterprise architecture design." IBM Systems Journal 43.3 (2004): 587-597.

4. Chen, David, Guy Doumeingts, and François Vernadat. "Architectures for enterprise integration and interoperability: Past, present and future." Computers in industry 59.7 (2008): 647-659.

5. Urbaczewski, Lise, and Stevan Mrdalj. "A comparison of enterprise architecture frameworks." Issues in Information Systems 7.2 (2006): 18-23. 
6. Schekkerman, J. "Enterprise Architecture Validation-Achieving Business-Aligned and Validated Enterprise Architectures." Institute For Enterprise Architecture Developments, URL: http://www. enterprisearchitecture. info 27 (2004).

7. Zachman, John A. "The concise definition of the Zachman framework." Zachman International (2008).

8. Lankhorst, Marc. Enterprise architecture at work. Vol. 352. Berlin: Springer, 2009.

9. Beznosov, Konstantin. "Information enterprise architectures: problems and perspectives." Written for the Advanced Topics in Software Engineering seminar given by Dr. Michael Evangelist at the School of Computer Science, Florida International University. 2000.

10. Goethals, Frank G., et al. "Management and enterprise architecture click: The FAD (E) E framework." Information Systems Frontiers 8.2 (2006): 67-79.

11. TOG (2013). Version 9.1. The Open Group. Available at: http://www.opengroup.org/togaf/. Accessed: June 2018.

12. Sessions, Roger. "A comparison of the top four enterprise-architecture methodologies." Houston: ObjectWatch $\operatorname{Inc}(2007)$.

13. Schneider, Alexander W., Christopher Schulz, and Florian Matthes. "Goals in Enterprise Architecture Management-Findings from Literature and Future Research Directions." 2013 IEEE 15th Conference on Business Informatics. IEEE, 2013.

14. Urbaczewski, Lise, and Stevan Mrdalj. "A comparison of enterprise architecture frameworks." Issues in Information Systems 7.2 (2006): 18-23.

15. Wegmann, Alain. The systemic enterprise architecture methodology (seam). business and it alignment for competitiveness. No. REP_WORK. 2002.

16. Regev, Gil, et al. "A philosophical foundation for business and IT alignment in enterprise architecture with the example of SEAM." Proceedings of the Third International Symposium on Business Modeling and Software Design. No. CONF. SCITEPRESS-Science and Technology Publications, 2013.

17. Golnam, Arash, et al. "Designing value-oriented service systems by value map." International Symposium on Business Modeling and Software Design. Springer, Cham, 2013.

18. Dahalin, Zulkhairi Md, et al. "An enterprise architecture methodology for business-it alignment: adopter and developer perspectives." Designing Enterprise Architecture Frameworks(2010): 229.

19. DoD (2013). DoDAF. Systems \& Software, Consortium. Available at: www.software.org/pub/architecture/dodaf.asp. Accessed: March 2018.

20. Dryer, David A., et al. "DoDAF limitations and enhancements for the Capability Test Methodology." Proceedings of the 2007 spring simulation multiconference-Volume 3. Society for Computer Simulation International, 2007.

21. Coleman, Preston, and Raymond Papp. "Strategic alignment: analysis of perspectives." Proceedings of the 2006 southern association for information systems conference. 2006.

22. Klein, John, and Michael Gagliardi. A workshop on analysis and evaluation of enterprise architectures. No. CMU/SEI2010-TN-023. CARNEGIE-MELLON UNIV PITTSBURGH PA SOFTWARE ENGINEERING INST, 2010.

23. Chapurlat, Vincent, and Christian Braesch. "Verification, validation, qualification and certification of enterprise models: Statements and opportunities." Computers in Industry 59.7 (2008): 711-721.

24. Kaplan, Robert S., and David P. Norton. "Transforming the balanced scorecard from performance measurement to strategic management: Part I." Accounting horizons 15.1 (2001): 87-104.

25. Abdullah, Iqra, et al. "Developments on balanced scorecard: a historical review." World Applied Sciences Journal 21.1 (2013): 134-141.

26. Van Grembergen, Wim, and Ronald Saull. "Aligning business and information technology through the balanced scorecard at a major Canadian financial group: its status measured with an IT BSC maturity model." Proceedings of the 34th annual Hawaii international conference on system sciences. IEEE, 2001.

27. Ylimäki, Tanja, and Veikko Halttunen. "Method engineering in practice: A case of applying the Zachman framework in the context of small enterprise architecture oriented projects." Information Knowledge Systems Management 5.3 (2005): 189-209.

28. Rehkopf, Thomas W., and Nicholas Wybolt. "Top 10 architecture land mines [enterprise]." IT professional 5.6 (2003): 36-43.

29. Rudawitz, D. "Why Enterprise Architecture Efforts Often Fall Short." Antevorte Consulting, LLC (2003).

30. Van Grembergen, Wim, and Ronald Saull. "Information technology governance through the balanced scorecard." Information technology evaluation methods and management. IGI Global, 2001. 199-211.

31. Morganwalp, Jillian M., and Andrew P. Sage. "Enterprise architecture measures of effectiveness." International Journal of Technology, Policy and Management 4.1 (2004): 81-94.

32. Stanley, Ronald, and Lorna Uden. "Why projects fail, from the perspective of service science." 7th international conference on knowledge management in organizations: service and cloud computing. Springer, Berlin, Heidelberg, 2013.

33. IEEE (1998). IEEE standard for software test documentation. Version 7, 1998. New York: IEEE - 1998. IEEE (1998). Test Case Specification Template, Test Case Specification Identifier, (IEEE 829-1998). IEEE (2000). IEEE Recommended Practice for Architectural Description of Software Intensive Systems. IEEE Standard 1471-2000.

34. Van Der Raadt, Bas, Sander Schouten, and Hans Van Vliet. "Stakeholder perception of enterprise architecture." European Conference on Software Architecture. Springer, Berlin, Heidelberg, 2008.

35. Baker, D. C., and M. Janiszewski. "Essential Elements of EA-Follow this roadmap to engage your business, manage complexity, and govern the implementation of your architecture by." Diamond-management and technology consultants (2005). 
36. Lam, Wing. "Investigating success factors in enterprise application integration: a case-driven analysis." European journal of information systems 14.2 (2005): 175-187.

37. Curran, Chris. "Link IT Investments to Business Metrics." Enterprise Architect 3.1 (2005): 16-18.

38. Gangemi, Aldo, et al. "Modelling ontology evaluation and validation." European Semantic Web Conference. Springer, Berlin, Heidelberg, 2006.

39. McGuinness, D. L., \& Van Harmelen, F. (2004). OWL web ontology language overview. W3C recommendation, 10(10), 2004.

40. Di Maio, Paola. "'Just enough'ontology engineering." arXiv preprint arXiv:1108.1488 (2011).

41. Stvilia, Besiki. "A model for ontology quality evaluation." First Monday 12.12 (2007).

42. Dutoit, Allen H., et al. "Rationale management in software engineering: Concepts and techniques." Rationale management in software engineering. Springer, Berlin, Heidelberg, 2006. 1-48.

43. Green, Nigel, and Carl Bate. Lost in translation: A handbook for information systems in the 21st century. New York: Evolved Technologist Press, 2007.

44. Oussena, Samia, and Joe Essien. "Validating enterprise architecture using ontology-based approach: A case study of student internship programme." 2013 3rd International Symposium ISKO-Maghreb. IEEE, 2013. 